\title{
LIGHT-CONTROLLED TRANSPORT IN DOPED DILUTED MAGNETIC SEMICONDUCTORS NEAR LOCALIZATION BOUNDARY*
}

\author{
P. Glód, T. Dietr, T. Wojtowicz, M. SAWicki \\ Institute of Physics, Polish Academy of Sciences \\ Al. Lotników 32/46, 02-668 Warszawa, Poland
}

\section{AND I. MiotKowski}

Department of Physics, Purdue University, West Lafayette, IN 47907, USA

\begin{abstract}
We report millikelvin studies of light induced metastable changes of the conductivity of the In doped $\mathrm{Cd}_{0.95} \mathrm{Mn}_{0.05} \mathrm{Te}_{0.97} \mathrm{Se}_{0.03}$ crystals in the vicinity of the metal-insulator transition.
\end{abstract}

PACS numbers: 71.30.th, 75.50.Pp, 72.40.+w

In the studies of the metal-insulator transition (MIT) the control of a certain parameter, such as electron concentration $n$, or external conditions, such as magnetic field $B$, are of great importance. Unusual properties of diluted magnetic semiconductors (DMS), particularly the giant negative magnetoresistance, have made it possible to tune MIT by the magnetic field [1], and thus to examine quantitatively the behavior of transport phenomena in the vicinity of MIT critical point. The obtained results referred to the case of the high magnetic fields, in which electrons resided in one spin subband and Mn spins were spin polarized. With no doubt, the exploration of the critical region in DMS in the absence of the external magnetic field, as well as the study of $3 \mathrm{D}(B, n, \sigma)$ diagram of MIT would be equally important. Such an opportunity is offered by the recently discovered photo-memory effect in some doped $n$-type II-VI semiconductors [3-5, 9]. This photo-memory effect can serve as a tuning mechanism of the transition, as was demonstrated for GaAlAs [2].

Previous transport [3, 4] far infrared (FIR) absorption [4] and SQUID magnetization experiments [5] performed for In and $\mathrm{Ga}$ doped $\mathrm{Cd}_{1-x} \mathrm{Mn}_{x} \mathrm{Te}$ and $\mathrm{Cd}_{1-x} \mathrm{Mn}_{x} \mathrm{Te}_{1-y} \mathrm{Se}_{y}$ crystals on insulator side of MIT showed persistent increase in the concentration of shallow donors after illumination of the sample at low temperature. In the FIR experiment strong persistent increase in absorption due to impurity and optical hopping transitions, as well as the appearance of the electric-dipole

*This work was supported in part by grant no. 204669101 and 204759101 of the Committee for Scientific Research and by Maria Skłodowska-Curie Found through grant no. PAN/NSF-92-113. 
spin resonance of donor bound electrons was observed. Analysis of the temperature dependence of the photoionization cross-section, as measured via transport experiments, indicates that the defect responsible for the persistent photoconductivity is strongly coupled to lattice vibrations, and the large lattice relaxation mechanism [6] was found as the mechanism responsible for the observed metastability [7]. It was also concluded [9] that the photo-memory effect is connected with metastable nature of the In dopant which forms centers similar to the $\mathrm{Cl}$ and $\mathrm{In}$ donors in $\mathrm{CdZnTe} \mathrm{[8]} \mathrm{and} \mathrm{to} \mathrm{DX} \mathrm{center} \mathrm{in} \mathrm{GaAlAs.} \mathrm{Moreover,} \mathrm{magnetization} \mathrm{measurements}$ provided a support for the negative $U$ model for DX centers in II-VI semiconductors. Assuming that the ground state of the center is magnetically inactive, as it binds two electrons in a singlet state, a good agreement between the light induced persistent changes in magnetization and theoretical values calculated for bound magnetic polarons formed on shallow donors was found.

In this paper we present results of millikelvin studies of light induced metastable changes of the conductivity in the similar In doped $\mathrm{Cd}_{0.95} \mathrm{Mn}_{0.05} \mathrm{Te}_{0.97} \mathrm{Se}_{0.03}$ crystals grown by the Bridgman method. Bar-shaped samples were prepared by mechanical polishing followed by etching in $5 \%$ solution of $\mathrm{Br}_{2}$ in $\mathrm{CH}_{3} \mathrm{OH}$. Then six ohmic contacts were prepared by the soldering In to the fresh surface. Electron

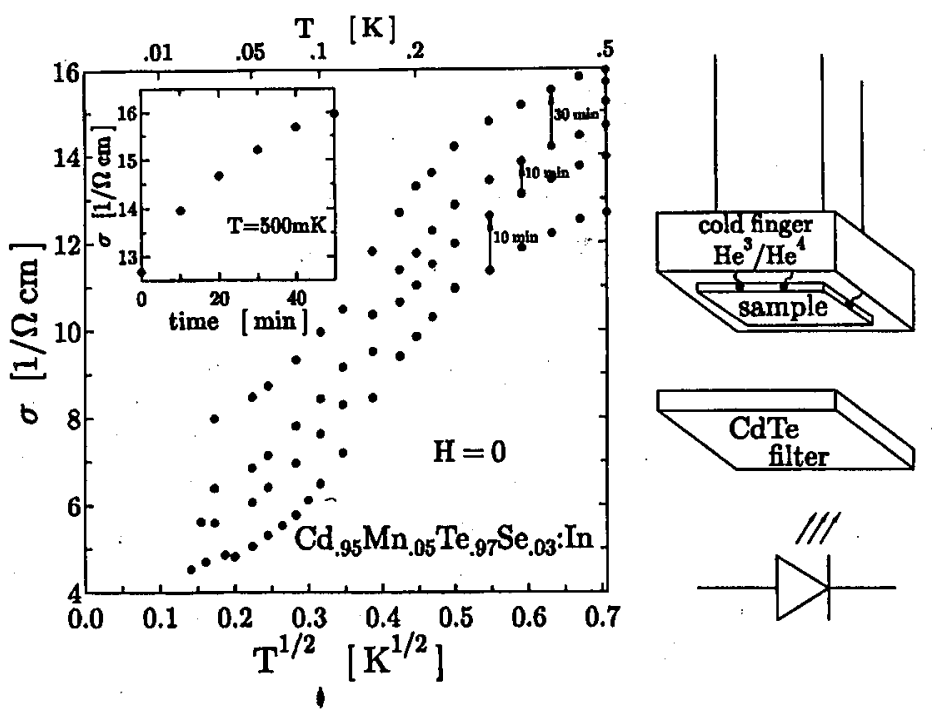

Fig. 1. Temperature dependence of the conductivity $\sigma$ after successive illumination steps for $\mathrm{Cd}_{0.95} \mathrm{Mn}_{0.05} \mathrm{Te}_{0.97} \mathrm{Se}_{0.03}$ :In near the metal-to-insulator transition. Inset shows tendency of $\sigma$ towards saturation after sufficiently long time of illumination. Right part of the figure shows schematically experimental arrangement for the measurement of the persistent change of conductivity in the dilution refrigerator. 


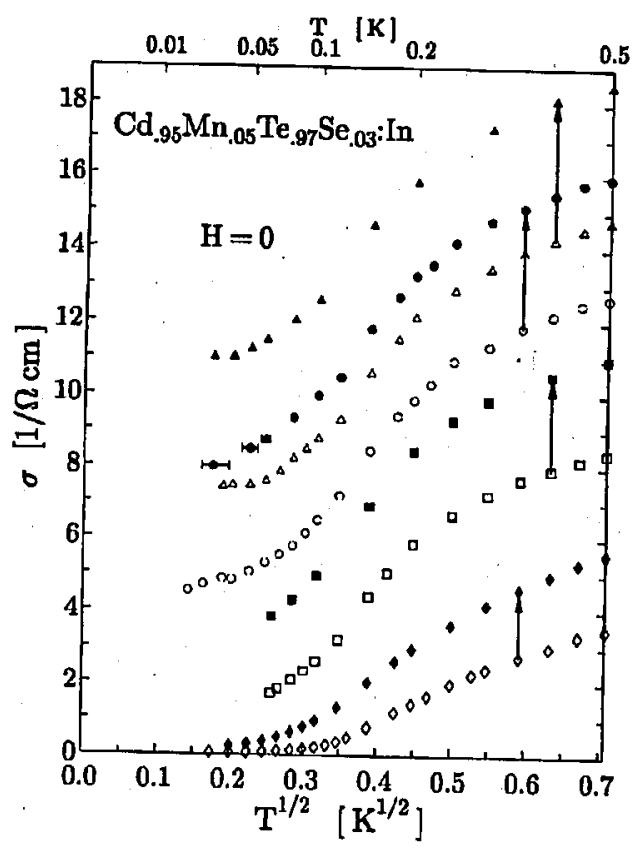

Fig. 2. Temperature dependence of conductivity for $\mathrm{Cd}_{0.95} \mathrm{Mn}_{0.05} \mathrm{Te}_{0.97} \mathrm{Se}_{0.03}$ : In with different electron concentrations, from $1.8 \times 10^{17}$ to $3 \times 10^{17} \mathrm{~cm}^{-3}$, on both sides of MIT. Arrows indicate the changes of $\sigma$ after long, close to saturation, illumination time (conductivities before and after illumination are marked with open and solid symbols respectively).

concentration of studied samples varied from $1.8 \times 10^{17}$ to $3 \times 10^{17} \mathrm{~cm}^{-3}$, as deduced from the Hall data at $300 \mathrm{~K}$, and were close to the critical concentration of MIT. Our measurements were performed in a dilution refrigerator (down to $30 \mathrm{mK}$ ) using a.c. technique (Ithaco 393 lock-in amplifier). The sample was pressed to the cold finger with the use of quartz plate. In order to get a uniform electron concentration, the light emitted from the diode was transmitted through a $\mathrm{CdTe}$ filter, so that only photons with energy below $E_{\mathrm{g}}$ of CdMnTeSe reached the sample.

The results of our studies are presented in Figs. 1 and 2. These figures show that the conductivity $\sigma$ of $\mathrm{Cd}_{0.95} \mathrm{Mn}_{0.05} \mathrm{Te}_{0.97} \mathrm{Se}_{0.03}$ :In crystals strongly decreases with decreasing temperature. This temperature dependent localization was observed previously by us in $\mathrm{Cd}_{1-x} \mathrm{Mn}_{x}$ Se:In $[10,11]$, and attributed to the formation of bound magnetic polarons. This interpretation was confirmed by a study of the temperature dependence of the resistivity $\rho$ of $\mathrm{Cd}_{1-x} \mathrm{Mn}_{x}$ Se:In for different Mn concentrations [12], which showed proportionality between resistivity and magnetic susceptibility, expected for scattering of diffusive electrons by bound magnetic polarons.

In all our data there is a visible tendency of $\sigma$ to saturation below $100 \mathrm{mK}$. Although we do not have any explanation for this phenomenon yet, we want to 
point out that this temperature is close to the spin glass freezing temperature. For temperatures below $100 \mathrm{mK}$ we observed also hysteresis in magnetic field dependencies of $\sigma$.

As can be seen in Figs. 1 and 2 we were able to observe light induced persistent change in electron concentration for samples on the both sides of MIT. The changes in the electron concentration induced in each of this samples were however not sufficient to induce MIT and therefore we were not able to determine the values of the relevant critical exponents. Nevertheless, by studying the temperature dependence of the conductivity after successive illumination steps in different samples we were able to trace temperature dependent localization across the metal-insulator transition. The obtained data contradict suggestions that the polaron formation could result in a discontinuous transition [13].

Further experiments on samples with higher light induced persistent change in the concentration of donors, which probably could be achieved after proper annealing process, are planned.

\section{References}

[1] T. Wojtowicz, T. Dietl, M. Sawicki, W. Plesiewicz, J. Jaroszyński, Phys. Rev. Lett. 56, 2419 (1986).

[2] S. Katsumoto, F. Komori, N. Sano, S. Kobayashi, J. Phys. Soc. Jpn. 56, 2259 (1987).

[3] I. Terry, T. Penney, S. von Molnar, P. Becla, Phys. Rev. Lett. 69, 1800 (1992); I. Terry, S. von Molnar, A.M. Torressen, P. Becla, Philos. Mag. B 65, 1245 (1992).

[4] T. Wojtowicz, N. Semaltianos, P. Kłosowski, M. Dobrowolska, J.K. Furdyna, I. Miotkowski, Acta Phys. Pol. A 80, 287 (1991).

[5] T. Wojtowicz, S. Koleśnik, I. Miotkowski, J.K. Furdyna, Phys. Rev. Lett. 70, 2317 (1993).

[6] J.M. Langer, Rev. Solid State Sci. 4, 229 (1990).

[7] N.G. Semaltianos, G. Karczewski, T. Wojtowicz, J.K. Furdyna, Phys. Rev. B, to be published.

[8] K. Khachaturyan, M. Kaminska, E.R. Weber, P. Becla, R.A. Street, Phys. Rev. B 40, 6304 (1989).

[9] I. Terry, T. Penney, S. von Molnár, J.M. Rigotty, P. Becla, Solid State Commun. 84, 235 (1992).

[10] T. Dietl, J. Antoszewski, L. Swierkowski, Physica B 117-118, 473 (1983).

[11] M. Sawicki, T. Dietl, J. Kossut, J. Igalson, T. Wojtowicz, W. Plesiewicz, Phys. Rev. Lett. 56, 508 (1986); T. Dietl, M. Sawicki, T. Wojtowicz, J. Jaroszyński, W. Plesiewicz, L. Świerkowski, J. Kossut, in: Anderson Localization, Eds. T. Ando, H. Fukuyama, Springer, Berlin 1988, p. 58.

[12] P. Głód et al., to be published.

[13] M.H. Cohen, E.N. Economou, C.M. Sokoulis, Phys. Rev. Lett. 51, 1202 (1983). 